\title{
Bone mineral density in postmenopausal women with high levels of parathyroid hormone
}

Irfan Esen, Selin Akturk Esen, Soner Cander, Ozen Oz Gul, Erdinc Erturk

Bursa Sevket Yilmaz Education and Reserach Hospital, Uludag University Medical School, Endocrinology and Metabolism

\section{Objectives}

- One of the main problems encountered with increased levels of serum PTH is decrease in bone mineral density in the long term.

- Decrease in bone mineral density is seen not only in hypercalcemic patients with primary hyperparathyroidism and also seen in normocalcemic patients with primary hyperparathyroidism.

- Secondary hyperparathyroidism due to vitamin D deficiency leads to mineralization disorders of the bones, low bone mineral density, osteoporosis and ultimately an increased risk of bone fracture in adults.

- In this study, we aimed to determine the reasons of secondary hyperparathyroidism for bone mineral density.

\section{Methods}

- One hundred and thirteen postmenopausal women with high serum PTH levels and 43 ones with normal PTH were enrolled to the study. Serum PTH level higher than $68.3 \mathrm{pg} / \mathrm{ml}$ was considered as hyperparathyroidism.

- Premenopausal women aged $<50$ years, postmenopausal women aged $\geq 75$ years, women with parathyroid gland disease, chronic renal failure, malabsorption, biphosphonate, thiazid or corticosteroid drug use and acute infection were excluded.

- Bone mineral density measurements in all participants were detected by dual-energy x-ray absorptiometry (DEXA) method using with Hologic QDR-4500A S/N 45130 analyzer (Hologic Inc. Bedford, MA, USA). The measurements of the lumbar spine and the left femur were recorded. The mineral density $\left(\mathrm{g} / \mathrm{cm}^{2}\right)$ of the second lumbar $(\mathrm{L})$ vertebra and the average mineral density of the L 1-4 vertebrae were compared statistically. Also the mineral density $\left(\mathrm{g} / \mathrm{cm}^{2}\right)$ of the left femoral neck and the average mineral density of the femoral neck, trochanteric and intertrochanteric regions were compared.

\section{Results}

The average age was $56.9 \pm 0.5(50-74, \mathrm{n}=113)$ in patients with hyperparathyroidism and $57.9 \pm 0.7(50-72, \mathrm{n}=43)$ in the control group. There were no significant differences between the groups in terms of menopausal age and body mass index (Table 1).

There was no significant difference between groups in terms of serum calcium levels but serum phosphorus levels were significantly lower in the study group. 24-hour urinary calcium excretion was reduced in the study group and 24-hour urinary phosphorus excretion was not different from the control group. Serum 25-OH vitamin D levels were low in both groups but there were no statistically significant differences between groups (Table 2).
The study group and the control group were compared in terms of bone mineral density (Table 3). The mineral density of the second lumbar vertebra and the average mineral density of the L 1-4 vertebrae were compared statistically. Also the mineral density of the left femoral neck and the average mineral density of the femoral neck, wards triangle and trochanteric region were compared. There was no significant difference in both regions in terms of bone mineral density. However, when all cases were evaluated together, the average mineral density of the L 1-4 vertebrae showed a reduction in parallel with the increase in serum PTH levels. It was statistically significant $(r=-0.175 ; \mathrm{p}=0.029)$ but the average mineral density of the femoral neck, wards triangle and trochanteric region did not show any increase or decrease in parallel with the increase in serum PTH levels.

\begin{tabular}{|c|c|c|c|c|}
\hline & $\begin{array}{c}\text { Study Group } \\
(\mathrm{n}=113)\end{array}$ & $\begin{array}{c}\text { Control Group } \\
(\mathrm{n}=43)\end{array}$ & & \\
\hline Age (year) & $56.9 \pm 0.5$ & $57.9 \pm 0.7$ & & \\
\hline Menopausal age (year) & $10.2 \pm 0.6$ & $9.7 \pm 0.9$ & & \\
\hline BMI $\left(\mathbf{k g} / \mathbf{m}^{2}\right)$ & $30.2 \pm 0.5$ & $29.4 \pm 0.5$ & & \\
\hline Creatinine (mg/dl) & $0.7 \pm 0.0$ & $0.6 \pm 0.1$ & & \\
\hline Chlorine (mmol/l) & $104.2 \pm 0.2$ & $104.2 \pm 0.3$ & 104 & \\
\hline ALP (IU/I) & $83.6 \pm 2.3$ & $81.3 \pm 3.1$ & & \\
\hline \multicolumn{5}{|c|}{ Data are given as mean $\pm \mathrm{SE}$ (standart error). ALP: alkaline phosphates, BMI: body mass index } \\
\hline \multicolumn{5}{|c|}{ Table-3: Comparison of the study group and the control group in terms of bone mineral density } \\
\hline & \multicolumn{2}{|c|}{ Study Group (n=113) } & Control Group (n=43) & $\mathrm{P}$ \\
\hline $\mathbf{L} 2\left(\mathbf{g} / \mathrm{cm}^{2}\right)$ & \multicolumn{2}{|c|}{$0.90 \pm 0.12$} & $0.89 \pm 0.12$ & 0.84 \\
\hline L1-L4 total $\left(\mathbf{g} / \mathrm{cm}^{2}\right)$ & \multicolumn{2}{|c|}{$0.90 \pm 0.12$} & & 0.36 \\
\hline Femoral neck $\left(\mathrm{g} / \mathrm{cm}^{2}\right)$ & \multicolumn{2}{|c|}{$0.75 \pm 0.11$} & & 0.68 \\
\hline Femur total $\left(\mathrm{g} / \mathrm{cm}^{2}\right)$ & \multicolumn{2}{|c|}{$0.95 \pm 0.01$} & & 0.97 \\
\hline
\end{tabular}

\begin{tabular}{|c|c|c|c|}
\hline & Study Group (n=113) & Control Group $(\mathrm{n}=43)$ & $\mathrm{P}$ \\
\hline Calcium (mg/dl) & $9.5 \pm 0.04$ & $9.5 \pm 0.05$ & 0.588 \\
\hline Adjusted calcium (mg/dl) & $9.6 \pm 0.04$ & $9.6 \pm 0.05$ & 0.355 \\
\hline Phosphorus (mg/dl) & $3.3 \pm 0.04$ & $3.6 \pm 0.7$ & $<0.001$ \\
\hline Creatinine clearance (ml/min) & $110.6 \pm 2.2$ & $108 \pm 3.5$ & 0.53 \\
\hline 24hUCa (mg/24s) & $132.3 \pm 7.3$ & $168.5 \pm 11.8$ & 0.006 \\
\hline $24 \mathrm{hUp}(\mathrm{mg} / 24 \mathrm{~s})$ & $637.6 \pm 23.4$ & $653.4 \pm 42.7$ & 0.956 \\
\hline TRp (\%) & $86.0 \pm 0.4$ & $86.7 \pm 0.6$ & 0.478 \\
\hline 25- OH vitamin D ( $(\mu \mathrm{g} / \mathrm{l})$ & $10.7 \pm 0.6$ & $12.1 \pm 1.3$ & 0.439 \\
\hline Uca /Ucr & $0.009 \pm 0.004$ & $0.012 \pm 0.004$ & 0.001 \\
\hline
\end{tabular}

24hUCa: 24-hour urinary calcium excretion, 24hUp: 24-hour urinary phosphorus excretion, TRp: tubular phosphorus reabsorption, Uca /Ucr: urinary calcium-creatinine ratio

\section{Conclusions}

- Decrease in bone mineral density was seen not only in patients with primary hyperparathyroidism and also seen in patients with secondary hyperparathyroidism due to vitamin D deficiency. Nakamura et al. (12) showed a significant decrease in bone mineral density in patients with hyperparathyroidism but they also showed a decrease in bone mineral density in patients with vitamin D deficiency independently from PTH levels. There are no adequate data about what levels of increase in serum PTH may adversely affect bone mineral density (13). Albettazzi et al. (5) compared the cases with elevated serum PTH levels and with normal serum PTH levels in their study but they could not detect a significant relationship between serum PTH levels and the bone mineral density of the lumbar spine and the femoral neck. Similarly, Kaya et al. (14) found no association between bone mineral density and elevated serum PTH levels in their study. But Silverberg et al. (15) demonstrated slightly decrease in bone mineral density with elevated PTH levels in their study. In our study, there is no significant difference between the study and control group in terms of bone mineral density and it may be related to the duration of hyperparathyroidism.

- Our study was a cross-sectional study and we did not know how long the patients had hyperparathyroidism. Probably, due to short-term exposure of elevated PTH levels and the small number of the study group, a significant difference could not be identified between the two groups in terms of bone mineral density. In addition, the levels of vitamin D deficiency in both groups were not very different. Therefore, it is considered that the levels of secondary hyperparathyroidism, the main factor that causes bone resorption, were not at the levels of negative effect on bone mineral density. 